\title{
REPRESENTATIONS OF INVARIANT MANIFOLDS FOR APPLICATIONS IN THREE-BODY SYSTEMS
}

\begin{abstract}
K. Howell, ${ }^{\star}$ M. Beckman, ${ }^{\dagger}$ C. Patterson, ${ }^{\star *}$ and D. Folta ${ }^{\S}$
The Lunar $L_{1}$ and $L_{2}$ libration points have been proposed as gateways granting inexpensive access to interplanetary space. To date, only individual solutions to the transfer between threebody systems have been found. The methodology to solve the problem for arbitrary three-body systems and entire families of orbits is currently being studied. This paper presents an initial approach to solve the general problem for single and multiple impulse transfers. Two different methods of representing and storing the invariant manifold data are presented. Some particular solutions are presented for two types of transfer problems, though the emphasis is on developing the methodology for solving the general problem.
\end{abstract}

\section{INTRODUCTION}

With the increasing interest in missions involving Sun-Earth and Earth-Moon libration points, it is necessary to further develop numerical, and possibly semi-analytical, tools to assist in trajectory design in multi-body regimes, including libration point orbits. Halo orbits are well-known examples of periodic orvits in such regions of space. Thus far, Earth-to-halo transfers, as well as halo-to-Earth arcs, have been computed using a number of different numerical procedures including exploitation of the invariant manifolds associated with a particular periodic halo orbit (or quasi-periodic Lissajous trajectory). More recently, transfers between different three-body systems are a new focus for potential mission scenarios. Developing transfers between Earth and a halo orbit or between different three-body systems involves numerically integrating trajectories from different initial conditions near a desired halo orbit manifold until one is identified that is most suitable for the application of interest. Of course, the stable/unstable invariant manifolds that correspond to a single halo orbit are infinite in number, but reside on the surface of a tube. ${ }^{1}$ Nevertheless, continuous computation of individual manifolds using numerical integration is not efficient or even practical for some applications. In the analysis of trajectories to/from a halo orbit, for example, the size of the most useful periodic orbit may be unknown and its amplitude may serve as a design parameter. The design space then includes not just a tube corresponding to the invariants manifolds of one halo orbit; rather it becomes a volume consisting of many tubes. For the problem of system-to-system transfers, the goal is the intersection of two manifold tubes - one from each system. A maneuver at an intersection point will shift the vehicle

\footnotetext{
Professor, School of Aeronautics and Astronautics, Purdue University, Grissom Hall, 315 North Grant St., West Lafayette, Indiana 47907-1282.

${ }^{\dagger}$ Flight Dynamics Engineer, Bldg 11, Room E121, NASA Goddard Space Flight Center, Greenbelt, Maryland 20771.

** Graduate Student, School of Aeronautics and Astronautics, Purdue University, Grissom Hall, 315 North Grant St., West Lafayette, Indiana 47907-1282.

${ }^{\S}$ Senior Flight Dynamics Engineer, Bldg 11, Room S116, NASA Goddard Space Flight Center, Greenbelt, Maryland 20771.
} 
from one tube to the other. In the Circular Restricted Three Body Problem (CR3BP), the flow in this region of space can also be visualized by noting that the tube is a separatrix, i.e., it bounds different regions of the flow. Thus, transit trajectories might be sought that pass inside the tubes and shift from one tube to the next. In this case, the spatial intersection of the tubes is still the key information to complete the computations. Computing those intersections is facilitated by efficient but accurate approximations of the tubes. So, the objective of this work is twofold: (i) representations/approximations of the invariant manifolds associated with periodic libration point orbits that are efficient and accurate in an automated process; (ii) demonstration that the approximation can be used to generate solutions to representative problems.

It is generally impossible to determine an analytical expression for stable and unstable manifolds so accurate numerical computations are required. This method usually proceeds by using one set of initial conditions and numerically integrating a single trajectory. Increasingly, the required trajectory length is long and accuracy suffers if the step size is small enough to accommodate potentially sharp changes along the path. Hobson offers some consideration of this issue and a method for approximating the manifold that includes an estimate of the errors. ${ }^{2}$ Hobson's method is an improved approach to globalize the manifold in terms of step size, but it still employs the standard first order approximation to initiate the process. A single trajectory may not, however, reflect the actual complex dynamical behavior of the system. In fact, the problems of interest here include a complete set of manifolds associated with a periodic orbit as part of the potential solution space. In configuration space, the stabie or unstabie manifolds are located along the surface of a tube corresponding to the periodic halo orbits. Either the numerical computation of some minimum number of orbits is required to represent the tubes or individual trajectories must be computed or recomputed as needed. If such data must be available for analysis, it may require a large amount of storage and retrieval capability. The specific requirements depend upon the application, of course. Relatively recently, a number of mathematicians have investigated approximation techniques. Guder et al. ${ }^{3}$ introduce cell mapping for the prediction of long term behavior and global analysis of nonlinear dynamical systems. Dellnitz and Hohmann ${ }^{4}$ use these cell mapping techniques combined with a subdivision procedure to approximate unstable manifolds and global attractors. However, they are not interested in determining the full global behavior; rather a numerical method that allows the approximation of the global attractor up to a specified accuracy. A box in $\mathbb{I R}^{n}$ is specified in which the dynamical behavior is to be analyzed. The box is subdivided and boxes discarded that do not contain part of the relative global behavior. A continuation method is further developed by Dellnitz and Hohmann to approximate the types of invariant manifolds of interest here. This method is also called an "outer approximation" of the unstable manifold. It is very useful to obtain a global view, however, it does not offer a parameterization of the manifold via arc length and small details may cause difficulty. Another technique by Krauskopf and Osinga ${ }^{5,6}$ is designed to specifically produce a list of points on the manifold by adding new points at prescribed distances from the last point and essentially "growing" the manifold. It is difficult to search this type of solution space, however, for the state information that is desired for the applications presented in this paper.

Approximating the manifold tubes for application to mission design is the first step in this current investigation. The process must be straightforward and offer accurate representations of the position and velocity states. It might also be used to represent the volume that includes multiple manifold surfaces to aid in determining a specific halo orbit for a given scenario. This process is applicable to error analysis, recovery and design for contingencies. Recent studies suggest that these types of analyses would greatly benefit from another parameter such as halo orbit size; if the manifold is represented in an easily accessible form, it can be accomplished in a automated manner. ${ }^{7,8}$ 
Application of these approximations to the system-to-system problem of recent interest is also used to demonstrate the approach. As noted by Farquhar et al., ${ }^{9}$ the libration points may become a primary hub for future human activities in the Earth's neighborhood. The Sun-Earth $\mathrm{L}_{2}$ point is expected to be where a number of large astronomical observatories will be located. These $L_{2}$ telescopes may require human servicing and repair as the missions and hardware become more complex and expensive. Closer to Earth, the Earth-Moon $\mathrm{L}_{1}$ libration point may serve as the staging node for the missions to the Sun-Earth $\mathrm{L}_{2}$ point as well as the Moon, Mars, and the rest of the solar system. Folta et al. ${ }^{10}$ produce various trajectories to support the human servicing role and offer other examples as well. But, improved and automated schemes for computation of such designs will be necessary for efficiency and larger studies. Lo and Ross" also support the Earth-Moon $L_{1}$ point as a "portal" to move beyond the Earth's neighborhood. The manifold tubes are introduced by Lo and Ross as the basis for the design strategy to produce the trajectories to move between these systems. Koon et al. ${ }^{12}$ describe the concept in more detail for the Earth-Moon to Sun-Earth problem. It has also been examined in the Jovian system. ${ }^{13}$ In any case, intersections may ultimately be sought between many tubes from many different halo orbits in each system; the complexity forces a new look at the computations. Of course, this problem creates a very complicated solution space and some representation of this set of solutions is sought to facilitate an automated design procedure. This study examines both splines and a cell structure to represent the manifolds.

\section{MANIFOLD APPROXIMATIONS: SPLINES}

In order to consider the intersection of a single trajectory (or a group of trajectories) with a surface that defines an invariant manifold corresponding to some halo orbit, the manifold must be generated and stored in an accessible format. One method investigated is the use of splines. The existing algorithms within the MATLAB ${ }^{\mathrm{TM}}$ Spline Toolbox ${ }^{\mathrm{TM}}$ are incorporated.

The numerical manifold data set that corresponds to one periodic halo orbit in some arbitrary system is first generated using a variable step propagator and the differential equations that model the circular restricted three-body problem (CR3BP). Multiple individual trajectories along one tube are generated to produce a good approximation to the entire manifold surface. For this analysis, $N$ points along the halo orbit are identified and then globalized to generate $N$ manifolds identified with one tubular surface corresponding to a specified periodic halo orbit. The initial state vectors are propagated and state vectors are identified along each trajectory, each at the same time, that result in $N \times 6$ vector elements that together represent a manifold surface. This data set is placed in a grid for later computations. The grid is comprised of $N$ columns and $M$ rows ( $M$ number of integration points, each at the same time). Spline knots are placed along each trajectory at the location of each isolated state vector, measured in time from the initial state at the halo orbit. The spline function is generated from the knot sequence, $\xi_{j}$, and the polynomial coefficients, $c_{j i}$,

$$
p_{j}(x)=\sum_{i=1}^{k}\left(x-\xi_{j}\right)^{k-i} c_{j i}, j=1: n
$$

A bivariate manifold spline is then generated for a manifold surface corresponding to a single halo orbit. The bivariate spline function yields manifold position data for a given trajectory, $\eta$, or equivalently the phase angle or time of the originating halo orbit (a total of $N$ trajectories), and the time from the start of the manifold, $\tau$ (a total of $M$ points), 


$$
\left[\begin{array}{l}
x \\
y \\
z
\end{array}\right]=c s(\eta, \tau)=\sum_{u=1}^{N} \sum_{v=1}^{M} p_{u}(\eta) p_{v}(\tau)
$$

This process enables calculation of position components given two parameters: number of the trajectory (or trajectory tag number) and time from the periodic orbit. The result appears in Figure 1.

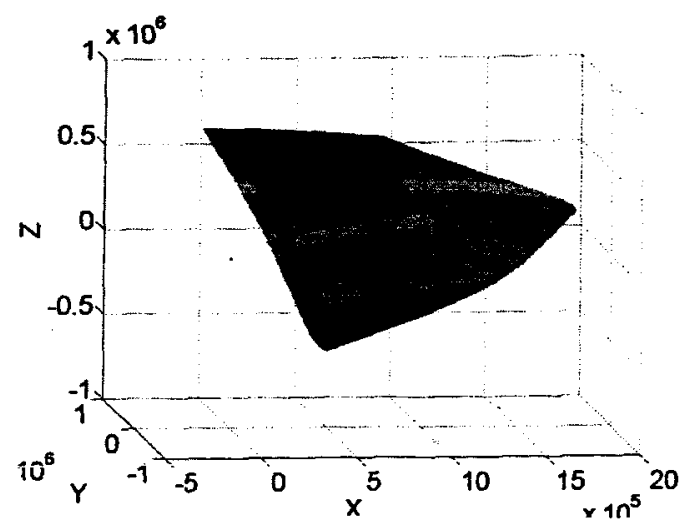

Sun-Earth $L_{1}$ (SEL1) Manifold

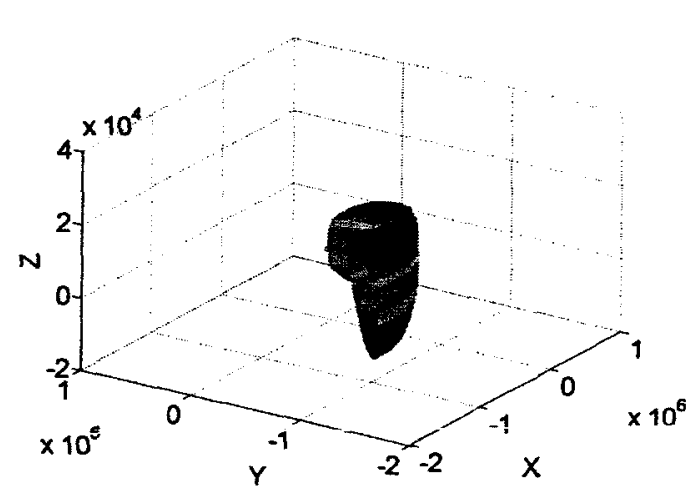

Earth-Moon $\mathrm{L}_{2}$ (EML2) Manifold

Figure 1 Position Splines

\section{MANIFOLDS APPROXIMATIONS: CELLS}

Also investigated is a representation of the manifolds in terms of a relatively straightforward cell structure such that each cell includes a polynomial model of one or more manifolds. Thus, the potential set of solutions may incorporate manifolds from various halo orbits. The cell structure then includes volume elements that can be searched for intersections with other single trajectories or intersections with other cells.

In the Sun-Earth system, consider a stable manifold tube that is computed numerically. With this initial surface, the manifold tubes that correspond to several additional, nearby halo orbits fill a volume in space as the individual manifold surfaces wrap around each other. To model any particular tube, exploit the fact that over small regions the stable manifold of a halo orbit in the circular restricted three-body problem is nearly flat and can be represented in position and velocity by low order functions. A volume of space through which several manifold surfaces pass and that is sufficiently small to be approximated in this manner is denoted here as a cell. (See Figure 2.) 

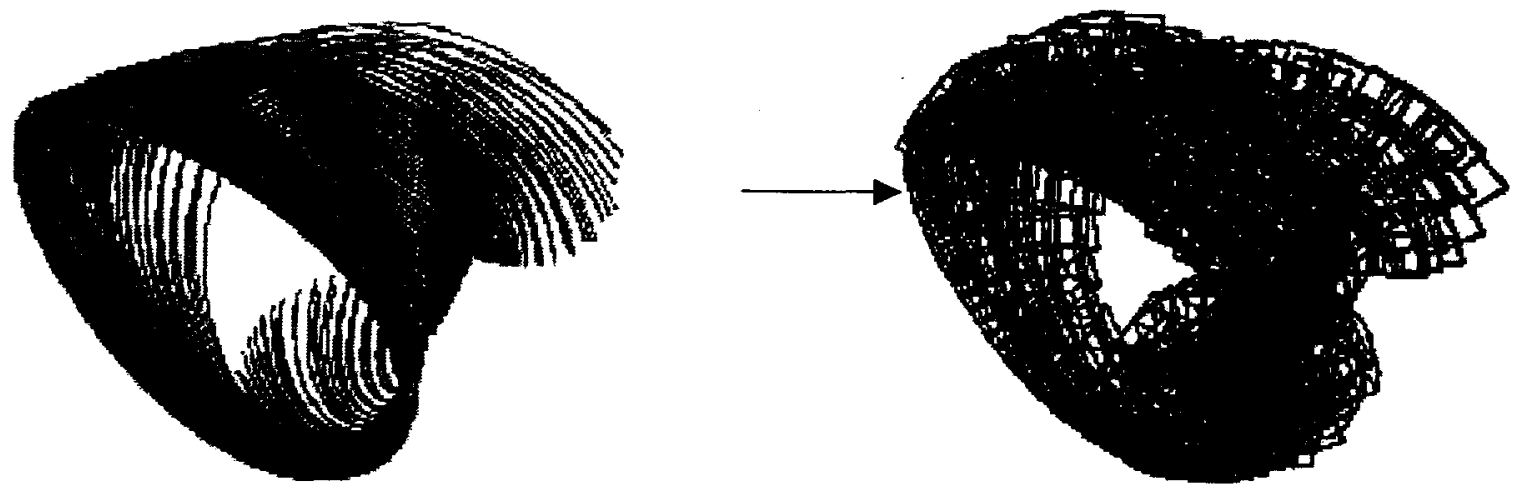

\section{Figure 2 Cells are Volumes in Space Defined to Contain Small Regions of Manifold Trajectories}

Within a cell, position data obtained from a single stable manifold tube are approximated using the $f$ it function from Mathematica ${ }^{\circ}$ to produce an analytical function of a surface. This process is repeated with other nearby manifolds until surface approximation functions for several manifolds passing through the cell are available. With this same position data, velocity data are also used to generate a single fit of each velocity component within the cell as a function of position. Thus, if $N$ stable halo manifolds pass through a single cell, $N$ fits to approximate the manifold surfaces are produced as well as $\mathrm{Nx} 3$ fits to approximate velocity components as a function of position.

\section{Fitting and Cell Shapes}

The velocity components are fit as third order polynomial functions of position in the standard rotating coordinate frame associated with the circular restricted three-body problem (CR3BP), that is,

$$
\begin{aligned}
\tilde{v}_{x, y, z}(x, y, z)= & a_{0}+a_{1} x+a_{2} x^{2}+a_{3} x^{3}+a_{4} y+a_{5} x y+a_{6} x^{2} y+ \\
& a_{7} y^{2}+a_{8} x y^{2}+a_{9} y^{3}+a_{10} z+a_{11} x z+a_{12} x^{2} z+ \\
& a_{13} y z+a_{14} x y z+a_{15} y^{2} z+a_{16} z^{2}+a_{17} x z^{2}+a_{18} y z^{2}+a_{19} z^{3}
\end{aligned}
$$

For the surface (position) fits, which often globally exhibit significant curvature, the position data are transformed to a spherical coordinate set and the surface is defined by a fit of radial data as a function of angular coordinates as follows,

$$
\begin{aligned}
\tilde{r}(\theta, \phi)= & a_{0}+a_{1} \cos (\phi)+a_{2} \cos ^{2}(\phi)+a_{3} \cos (\theta)+a_{4} \cos (\phi) \cos (\theta)+ \\
& a_{5} \cos ^{2}(\theta)+a_{6} \sin (\phi)+a_{7} \cos (\phi) \sin (\phi)+a_{8} \sin (\phi) \cos (\theta)+ \\
& a_{9} \sin ^{2}(\phi)+a_{10} \sin (\theta)+a_{11} \cos (\phi) \sin (\theta)+a_{12} \cos (\theta) \sin (\theta)+ \\
& a_{13} \sin (\phi) \sin (\theta)+a_{14} \sin ^{2}(\theta)
\end{aligned}
$$


where $\theta$ and $\phi$ are angles that orient the radial direction in 3D. The origin for the spherical set of coordinates is selected as the average center of curvature computed from every data point within the cell. This transformation is accomplished by defining unit vectors in the average tangential, normal, and binormal directions from the mean center of the data and placing the cell origin along the normal direction at a distance equal to the average radius of curvature. With a small data set, this choice encourages a cell shape that is indicative of the local shape of the manifold itself and a smooth surface fit with little variation in $r$. In some areas of the tube, however, manifold trajectories tend to lie in the same plane as the path representing the centers of curvature for the data; for a cell to contain several such trajectories this choice will result in a large variation in $r$. In this case, the origin is shifted to a location along the binormal direction. With the data points transformed to this frame, the cell boundaries are defined as the minimum and maximum values of $r, \theta$, and $\phi$. The fits are reasonably accurate everywhere except very near the Earth where curvature of the actual manifold is extremely high. Along the manifolds, a high curvature region requires the use of smaller cells within which the manifolds can be approximated as nearly flat. Also, the volume of space contained in a cell should not extend too far beyond the volume defined by the manifold. Consequently, cell sizes and shapes are tailored to the local shape of the manifold volume. Then, position and velocity functions for the manifold data within the cells can be determined with standard fitting algorithms. This process has been automated for examples of the type examined here. In Figure 3(a), the maximum and median position errors along the surfaces are compared with actual manifold tube data within the cells. Median errors are typically less than $500 \mathrm{~km}$, and maximum errors typically less than $5000 \mathrm{~km}$, the most notable exceptions being very near the Earth where the error actually extends beyond the range of the display. In Figure 3(b), the median and maximum percentage errors in the velocity functions are compared to actual velocity data. Note that the velocity errors are typically very small.
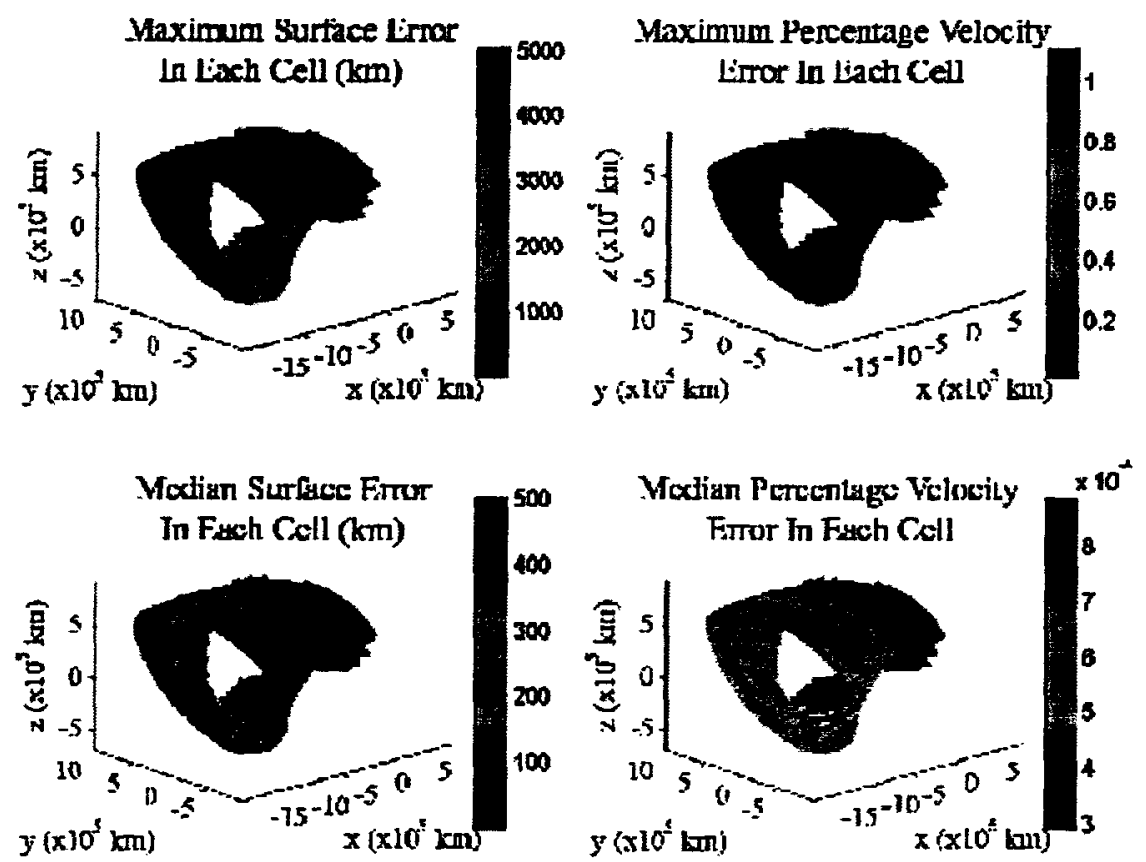

Figure 3 SEL1 Manifold Position and Velocity Fits :Halos Range in Size from $A z \approx 800,000 \mathrm{~km}$ to $\mathrm{Az} \approx 880,000 \mathrm{~km}$ 


\section{Cell Creation}

Approximation of an entire volume of manifolds involves slicing that volume into many such cells. The creation of the cells begins with a single tube of trajectories, each propagated backwards to its first periapsis point. The cells cover the entire area of the tube and are kept relatively small so that the analytical approximations remain accurate. Also, they are designed to overlap at their edges so that the fits within one cell mesh smoothly with the fits of a neighboring cell. Subsequently, the fits within the cells collectively represent a more continuous approximation of the manifold.

To create the cells, a manifold tube is sliced into two sections. The first section is formed by truncating the tube close to the periodic orbit. Using the libration point as an origin, each revolution along the path as it departs the orbit is sliced typically into 64 sectors and the data within each sector are used to define a new cell and frame as described above. This process is then repeated a predetermined number of times. The second section is comprised of the rest of the tube. The method of cell creation is then modified to more closely follow the evolution of the manifold trajectories. A limited number of neighboring trajectories on the manifold, denoted here as a "ribbon", is propagated backward from the last section to periapsis and sliced evenly in distance along its length. Each slice of data taken from the ribbon is used to define a new cell. The process is repeated using the last trajectory of the previous ribbon as the first trajectory of a new ribuon until the entire the tube is represented. Note that before this step is complete, some cells may require resizing to maintain accuracy. A maximum volume is selected and any cell larger than the maximum is sliced in half along its largest side. When placing data in cells it is also important that a minimum amount of data from a minimum number of trajectories always be present so that surface fits can be accurately made. To accomplish this, manifold tubes have actually been propagated twice, the second time containing three times the number of trajectories than the first, and with many more points. The manifolds with the sparse data are used for most of the cells created, but when the minimum point and trajectory requirements are not met within a cell, the densely populated arc is incorporated.

\section{Adding Manifold Data}

After initial cell creation, the cells contain the data corresponding to merely one manifold tube. Data from additional manifold tubes can be added with certain caveats. The cell shapes at this point are tailored to the original tube shape and size, so the trajectories of any other manifold tube will not be fully contained within their volumes. Therefore, to contain as much data as possible from a nearby tube, the cells may be required to increase in size. However, the fitting process is premised on the idea that manifold data can be approximated only over small regions, so cell growth is limited to encompass only the new tube data. A single cell originally contains all manifold data from one tube within a bounded volume. The data of a second tube is transformed to the original cell's spherical coordinate frame, and the angular bounds of the cell are increased a small amount. Any part of the new tube's data that fits within the new angular bounds of the cell is considered as data for a second surface within the cell. The bounds of the cell are then reset to reflect the increase in the volume of data stored. In Figure 4 is an example of three SEL1 manifold tubes which have been placed in the same set of cells. They range in size from $\mathrm{Az}=\sim 800,000 \mathrm{~km}$ to $\mathrm{Az}=\sim 880,000 \mathrm{~km}$. It is apparent that even with three tubes placed in the cells, there can be vacant areas. In these areas, the velocity fits will be less accurate. 
This problem can be solved by adding more manifold tubes without allowing cell growth, but maintaining the minimum number of points and trajectories. If, in a single cell, the minima are not met then the data for that surface does not get added to the cell. So some cells may contain more manifold tubes from a wider range of halos than other cells. It is also possible to approximate the wider range of halos by stacking cells, essentially implementing the cell creating process for the manifolds of one set of halos, then creating entirely new cells for a larger or smaller set and repeating.

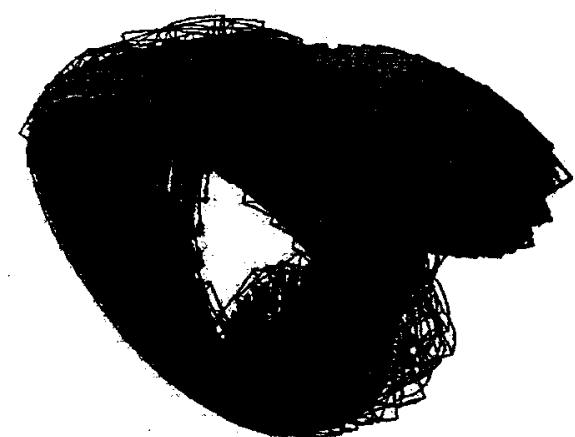

Figure 4 Five Tubes through a Set of Cells

\section{Example: Using Cells to Find Halo Transfers in the CR3BP}

With approximations defined for stable Sun/Earth $\mathrm{L}_{1}$ (SEL1) halo manifolds within the cells, the process of finding transfers to halo orbits is simplified. For any spacecraft trajectory that intersects a celi at a point $\mathbf{r}_{\text {int }}$ with a velocity $\mathbf{v}_{\text {int, }}$ an initial guess for a $\Delta \mathbf{V}$ that might be necessary to complete a transfer is simply $\Delta V=\tilde{V}\left(r_{\text {int }}\right)-\mathbf{v}_{\text {int } \text { t }}$ where $\tilde{V}\left(r_{\text {int }}\right)$ is the approximation for a manifold velocity at the intersection point. It is assumed that the continuous distribution of periodic orbits in a family possesses manifolds that exist on a continuum of surfaces passing through the cells, and that velocity on those surfaces varies continuously throughout the volume. Analytical approximations for only a few of the surfaces of position are planned to be available, but a single velocity approximation is continuous over the entire cell volume. So, even if $\mathbf{r}_{\text {int }}$ lies between or outside the known manifold surfaces within the cells, it is still identified with some member of the manifold continuum, thus, the velocity fit can be applied and used as an initial guess for the velocity of a manifold trajectory passing through that point. Moreover, it is not necessary that a particular halo orbit be specified in advance. Indeed, if the best intersection point within the cell is to be selected from among all possible points in the volume, and that best point is not known a priori, then any halo orbit in or near the range of those used to initially generate the manifolds and create the cells may serve as the ideal target halo for the transfer. So rather than selecting a halo and attempting to locate intersections with its stable manifold, an easier approach may be the determination of the intersections with a volume of stable manifolds that are associated with a set of periodic orbits and use the lowest-cost intersection point to define the target orbit.

\section{The Use of Surface Fits in Determining a Destination Halo}

An intersection point $\mathbf{r}_{\text {int }}$ inside a cell has spherical coordinates $(r, \theta, \phi)$. This point may be inside, outside, or somewhere between the surfaces defined within the cell that correspond to known manifold tubes as is apparent in Figure 5. To discover its location relative to the existing surface fits, calculate $\widetilde{r}_{i}(\theta, \phi)$ where $i$ ranges from 1 to $n$, the number of surfaces within the cell. Each surface, and thus each value of $\tilde{r}_{i}(\theta, \phi)$, is associated with a specific halo. These halo 


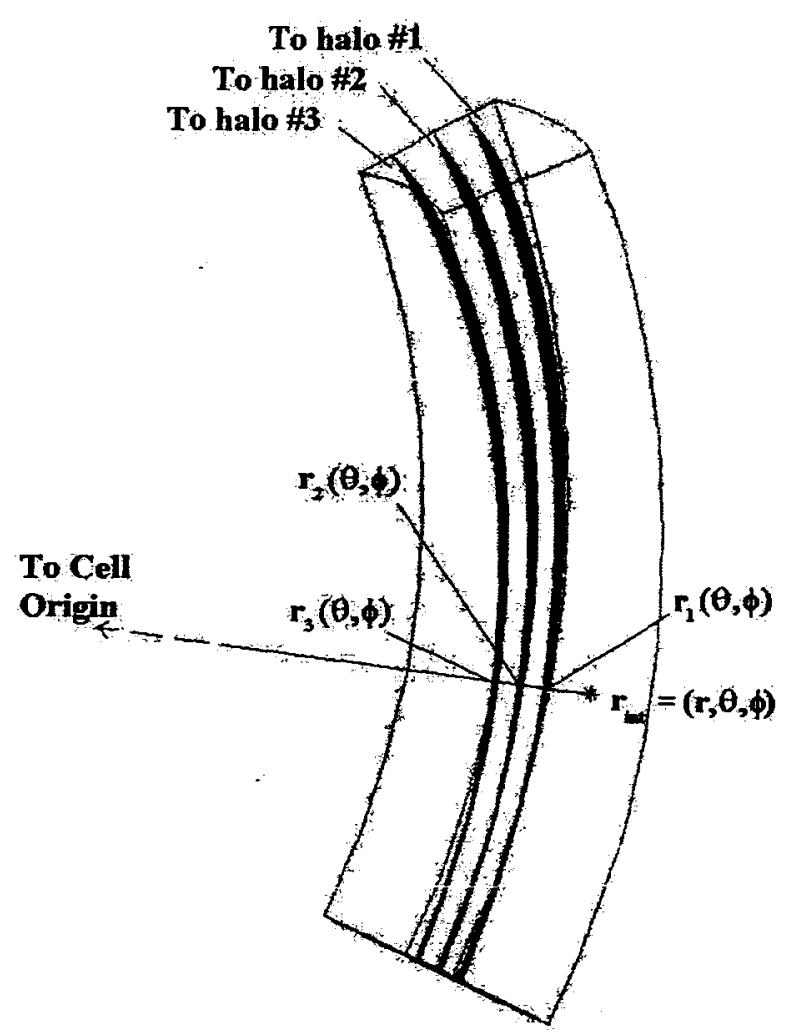

orbits can be characterized by the initial conditions used to propagate them numerically in the 3-body problem. For example, $\left(x_{0}^{i}, 0, z_{0}^{i}, 0, \dot{y}_{0}^{i}, 0\right)$ are the states at the point of the maximum $z$ amplitude and are used to parameterize a single halo orbit. Each initial condition is fit as a quadratic function of $\widetilde{r}_{i}(\theta, \phi)$, directly associating the halos to the sizes of the manifold tubes at $(\theta, \phi)$ within the cell. This procedure yields functions that can be solved for a set of initial conditions (and thus the size) of a destination halo orbit as a function of the location $r$ within the cell, that is,

$$
\begin{aligned}
& x_{0}=a_{x 0}+a_{x 1} r+a_{x 2} r^{2} \\
& z_{0}=a_{z 0}+a_{z 1} r+a_{z 2} r^{2} \\
& \dot{y}_{0}=a_{\dot{y} 0}+a_{\dot{y} 1} r+a_{\dot{y} 2} r^{2}
\end{aligned}
$$

Figure 5 Locating the Intersection Point within a Cell

The coefficients can be determined by solving the following three matrix equations,

$$
\begin{aligned}
{\left[\begin{array}{ccc}
1 & r_{1} & r_{1}^{2} \\
: & : & : \\
1 & r_{n} & r_{n}^{2}
\end{array}\right]\left\{\begin{array}{l}
a_{x 0} \\
\left.a_{x 1}\right\} \\
a_{x 2}
\end{array}\right\}=\left\{\begin{array}{c}
x_{0}^{1} \\
:\}, \\
x_{0}^{n}
\end{array}\right\}\left[\begin{array}{ccc}
1 & r_{1} & r_{1}^{2} \\
: & : & : \\
1 & r_{3} & r_{3}^{2}
\end{array}\right]\left\{\begin{array}{l}
a_{z 0} \\
\left.a_{z 1}\right\} \\
a_{z 2}
\end{array}\right\}=\left\{\begin{array}{c}
z_{0}^{1} \\
: \\
z_{0}^{n}
\end{array}\right\} } \\
{\left[\begin{array}{ccc}
1 & r_{1} & r_{1}^{2} \\
: & : & : \\
1 & r_{3} & r_{3}^{2}
\end{array}\right]\left\{\begin{array}{l}
a_{\dot{y} 0} \\
a_{\dot{y} 1} \\
a_{\dot{y} 2}
\end{array}\right\}=\left\{\begin{array}{l}
\dot{y}_{0}^{1} \\
: \\
\dot{y}_{0}^{n}
\end{array}\right\} }
\end{aligned}
$$


If the number of surfaces $n>3$, this is accomplished with a least squares fit. If $n<3$, then a less accurate linear fit must suffice. However, an effort has been made to always include at least three manifold surfaces in every cell.

\section{Approaching the Destination Halo}

Approximations from fit functions are generally most successful when applied in a region near or within the bounds of the region where the data are available. This region is defined by the surfaces within the cell. Thus, the surfaces nearest and furthest from the cell origin define the region. The distance between these two surfaces at any point $(\theta, \phi)$ in the cell defines a width, $w(\theta, \phi)$, and any trajectory intersection point that is more than a distance $w$ from the surfaces is ignored as a potential transfer point for the given cell (but, it would re-emerge in another cell). These low order velocity fits within the cells are generally sufficient to produce a trajectory arc from a point within the cell to the vicinity of the libration point orbit, but it is likely that it will not directly insert into a periodic orbit. The sensitivity of the problem demands that some amount of targeting is used to complete the transfer. However, with the approximation, it is an additional automated step that results in successful transfers to halo orbits in the circular restricted problem.

Assume that an initial trajectory originates from somewhere in or beyond the current system. There is no restriction on the source of the incoming leg. Assuming that this path will intersect one or more cells, they are identified and all intersecting points $\mathbf{r}_{\text {int }}$ within the cell are tagged. For each intersection point, the velocity discontinuity is calculated, that is, $\Delta \mathbf{V}_{\text {cell }}=\widetilde{\mathbf{V}}\left(\mathbf{r}_{\text {int }}\right)-\mathbf{v}_{\text {int. }}$ seeking the $\mathbf{r}_{\text {int }}$ that minimizes $\Delta \mathbf{V}_{\text {cell }}$. The calculated $\Delta \mathbf{V}_{\text {cell }}$ is applied. The point of closest approach to the halo is determined. Since $\Delta \mathbf{V}_{\text {cell }}$ is an approximation, the transfer arc will not approach the periodic orbit asymptotically. A halo orbit insertion (HOI) point is selected and targeted from $\mathbf{r}_{\text {int }}$ and a HOI $\Delta \mathrm{V}$ is included at the HOI point. This completes the transfer with a total cost $C=\left|k \Delta \mathbf{V}_{\text {cell }}+\Delta \mathbf{V}_{\text {targeter }}\right|+\left|\Delta \mathbf{V}_{\text {HOI }}\right|$ This cost is a function of the HOI point. For convenience, the MATLAB ${ }^{\mathrm{TM}}$ Optimization Toolbox ${ }^{\mathrm{TM}}$ function fminbnd is utilized to find a minimum of this cost as a function of the HOI location.

Numerical values for a test case appear in Table 1 and Figure 6. Three halos at $\mathrm{Az}=$ $\sim 800,000 \mathrm{~km}, \mathrm{Az}=\sim 840,000 \mathrm{~km}$ and $\mathrm{Az}=\sim 880,000 \mathrm{~km}$ are used to generate manifold cell volumes, and a fourth stable manifold to a halo of $\mathrm{Az}=\sim 945,000 \mathrm{~km}$, one that is not used in any of the fitting, is used to test the transfer procedure. A point along this actual stable manifold is perturbed with a $\Delta \mathbf{V}$ of $20 / \mathrm{ms}$ at a point within a cell. At this precise

Table 1

Targeting a Halo Orbit

$\begin{array}{cccc}\text { Step in Process } & \frac{\text { Destination }}{\underline{\text { Halo }}} & \frac{\text { Total } \Delta V \text { at }}{\text { intersection }} & \Delta \mathbf{V}_{\text {HOI }} \\ \begin{array}{c}\text { Exact Manifold } \\ \text { Intersection }\end{array} & \mathrm{Az}=945,481 \mathrm{~km} / \mathrm{s} & -- \\ \begin{array}{c}\text { Approximation Result } \\ \text { at Same Intersection Point }\end{array} & \mathrm{Az}=945,176 \mathrm{~km} & 19.69 \mathrm{~m} / \mathrm{s} & 1.43 \mathrm{~m} / \mathrm{s}\end{array}$


intersection point, the approximations within the cells are employed to estimate the velocity at this point, approximate the appropriate $\Delta \mathbf{V}$ and determine the specific halo that would minimize the cost. The actual manifold should be the best solution and the results of the approximations appear in the table. A good estimate of the destination halo is achieved and the transfer is accomplished with a cost very similar to the $20 \mathrm{~m} / \mathrm{s}$ perturbation.

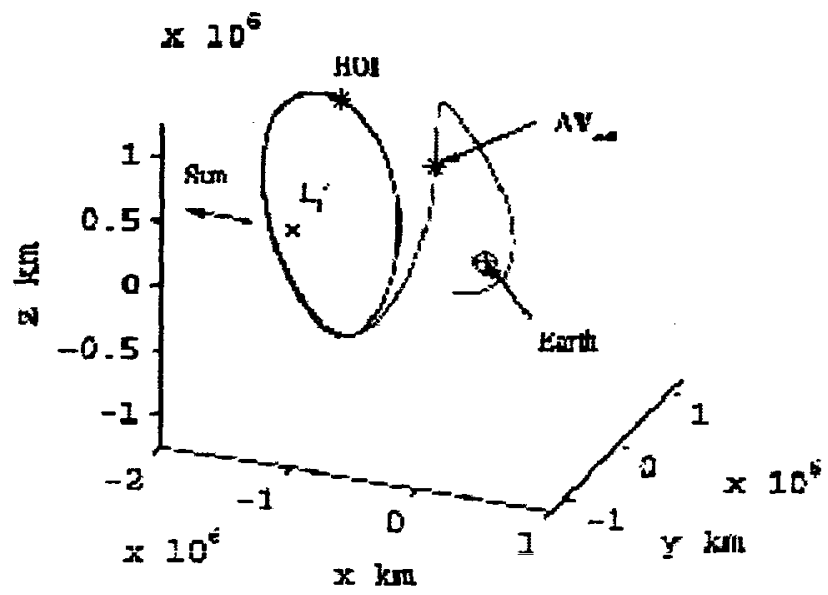

Figure 6 Transfer Determined Using Cells

\section{SYSTEM TO SYSTEM TRANSFERS}

A more challenging problem is the system to system transfer. In particular, for demonstration here, the transfer from the Earth-Moon system to the Sun-Earth system is considered. As noted previously, these transfers are the focus of a number of researchers. ${ }^{14}$ In this work, an efficient method of computation is sought using approximations to the manifolds. The ultimate objective is to use the approximation to produce the transfers in full, 3-D ephemeris models that may include other perturbations as well. This problem is approached using both splines and cell structures. Ultimately both may be useful in designing these types of trajectories.

\section{Using Splines to Determine the Intersections of Earth-Moon and Sun-Earth Tubes}

Using the manifold position spline functions, intersections can be computed between invariant manifolds of different three-body systems. Assuming that the goal is to shift directly from the surface of one manifold to the other, these intersections result in one-impulse transfers between the three-body systems. Such could be the case if it was desired to depart an Earth-Moon $\mathrm{L}_{2}$ orbit and reach a Sun-Earth $\mathrm{L}_{1}$ halo orbit, for example. Figure 7 includes an example of a large Sun-Earth $\mathrm{L}_{1}$ stable manifold and various Earth-Moon $\mathrm{L}_{2}$ unstable manifolds at different phases of the Moon. From this figure, it is apparent that the tubes intersect. Using a 


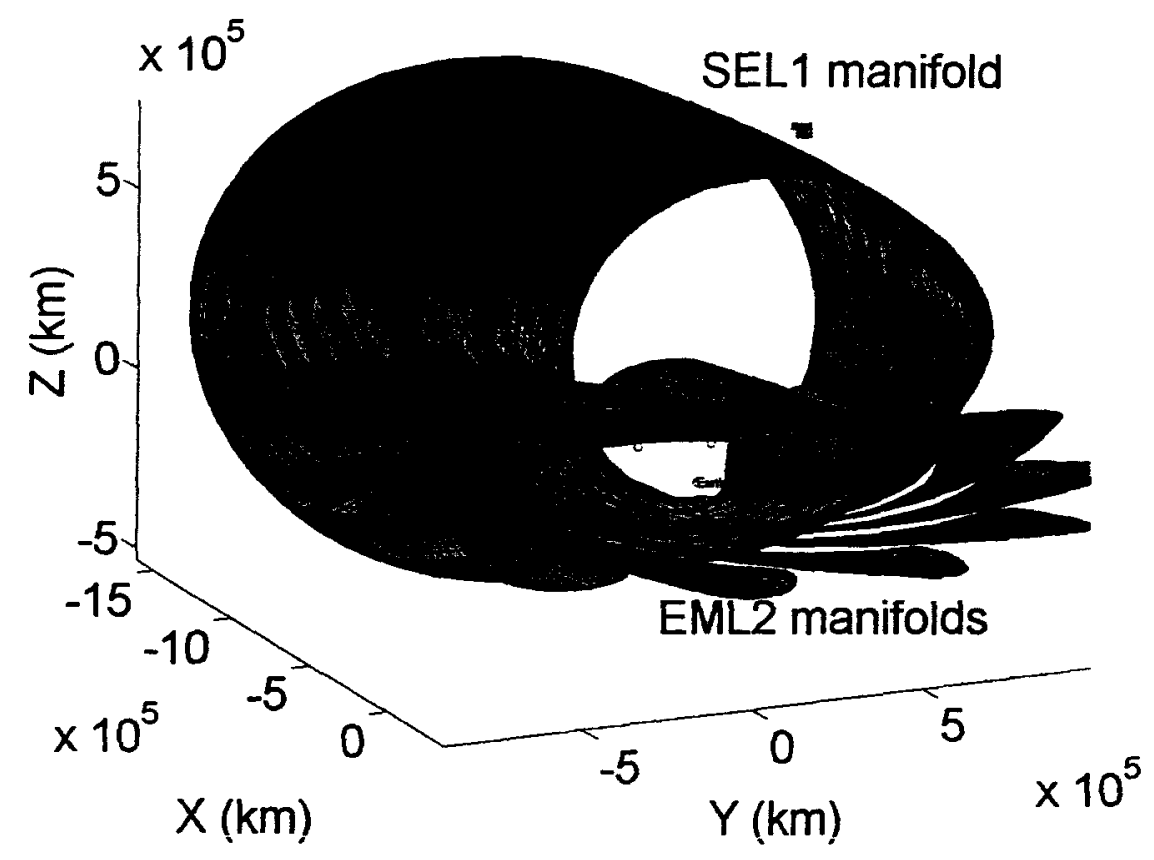

Figure 7 Intersections Between Tubes

Moon phase of $210 \mathrm{deg}$, an intersection between the manifolds is assured at greater than one point. The Matlab Optimization Toolbox is employed to determine the intersections. The Matlab function fmincon yields a minimum of a constrained nonlinear multivariate function. Mediumscale optimization is used and the gradient is determined through finite-differencing. The SQP method closely mimics Newton's method for constrained optimization just as is done for unconstrained optimization. At each major iteration, an approximation is made of the Hessian of the Lagrangian function using a quasi-Newton updating method. This Hessian is then used to generate a quadratic programming subproblem whose solution is used to form a search direction for a line search procedure. The solution yields a position on each manifold, the two of which differ by only $43 \mathrm{~km}$ for this example. The first successful use of fmincon gives us one point of intersection. It is necessary to find additional points of intersection where an entire set should be closed ring (assuming complete intersection of entire tube). Such a ring can be computed by adding additional constraints to fmincon. A nonlinear equality constraint forces fmincon to locate a second point of intersection between the two manifolds but at a distance $h$ from the first point. The distance $h$ is currently set at $1000 \mathrm{~km}$ as an initial guess. The ring is successfully computed and appears in Figure 8. Once the intersection is determined in configuration space, the velocity differences are also available, and the cost can be computed. 


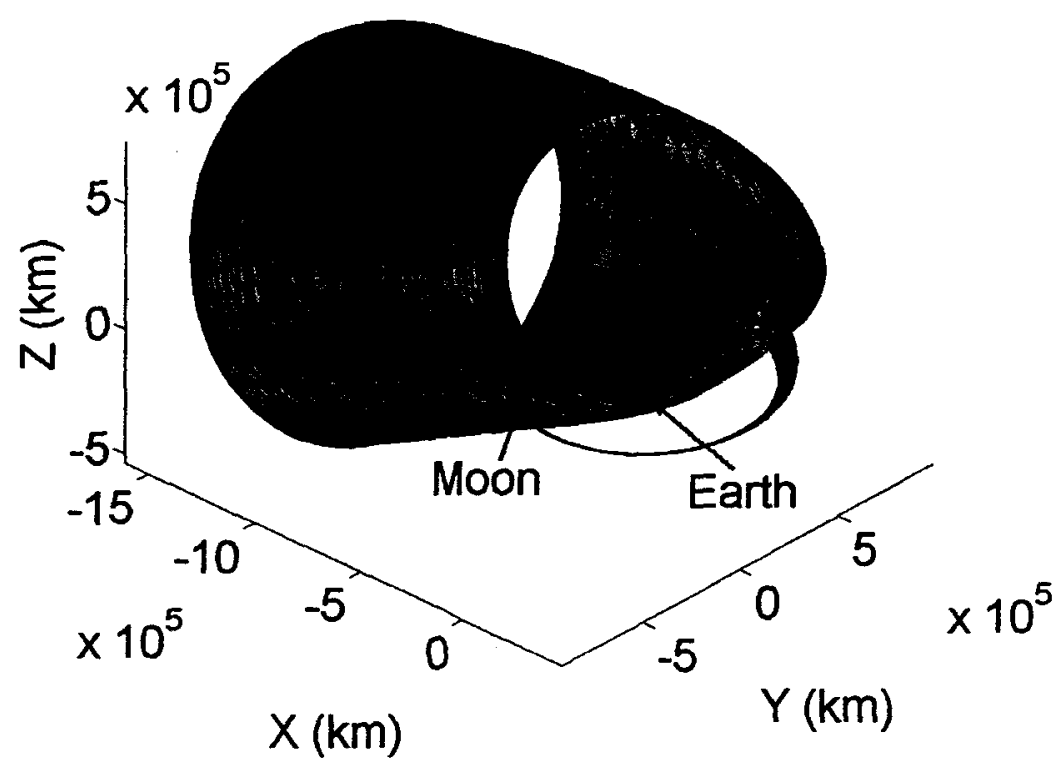

Figure 8 Ring of Intersection

\section{Using Cells to Approximate a Transfer and Shift into the Ephemeris Model}

The same Earth-Moon system to Sun-Earth system transfer was also attempted using the cell structure. In this case, the problem is solved in two steps. First, an approximate solution is obtained in the circular restricted problem using the cells. Then, the solution is transferred to a more complete model. Using the software package Generator, developed at Purdue University, this approximation is used to produce an end-to-end trajectory from an Earth-Moon libration point orbit to a Sun-Earth libration point orbit. It is noted that other researchers are also seeking transfers of this type in the full ephemeris model. ${ }^{10-12}$

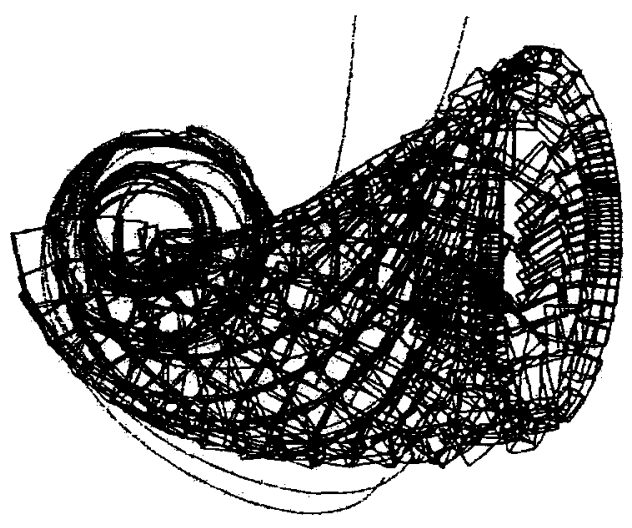

Figure 9 EML1 Paths Intersect Cells
In the circular restricted Earth-Moon system, unstable manifold trajectories for a $\mathrm{L}_{1}$ halo of amplitude $\mathrm{Az}=\sim 39,000 \mathrm{~km}$ are propagated. These manifolds form a tube, of course. Any of the trajectories can be transformed to Sun-Earth coordinates and intersections are determined with cells that correspond to the SEL2 stable manifold. A set of cells is generated for SEL2 stable manifolds to halos ranging from $\mathrm{Az}=\sim 170,000 \mathrm{~km}$ to $\mathrm{Az}=$ $\sim 220,000 \mathrm{~km}$ as seen in Figure 9. These cells contain position and velocity fits for the data of only three tubes. Initially, the Moon is $45^{\circ}$ out-of- 
phase with the Sun-Earth system. It is also noted that the EM frame is rotated $-5^{\circ}$ about the SunEarth $x$-axis to more accurately represent the inclination of the Moon's orbit. After the transformation to the Earth-Sun system, the Earth-Moon manifold trajectories are searched to locate all intersections with the SEL2 cells, and the transfer approximation process proceeds. In Figure 9, all EM manifold trajectories are overlaid with the SEL2 cell outlines. The estimated total cost for the "best" transfer is $24.518 \mathrm{~m} / \mathrm{s}$, as determined in the circular restricted problem. The transfer appears in Figure 10 in both the circular restricted Earth-Moon view (Figure 10(a)) and the circular restricted Sun-Earth view (Figure 10(b)). In the Earth-Moon view it is observed that the manifold that departs $L_{1}$ does encircle $L_{2}$ as it departs the system. This characteristic is actually familiar.

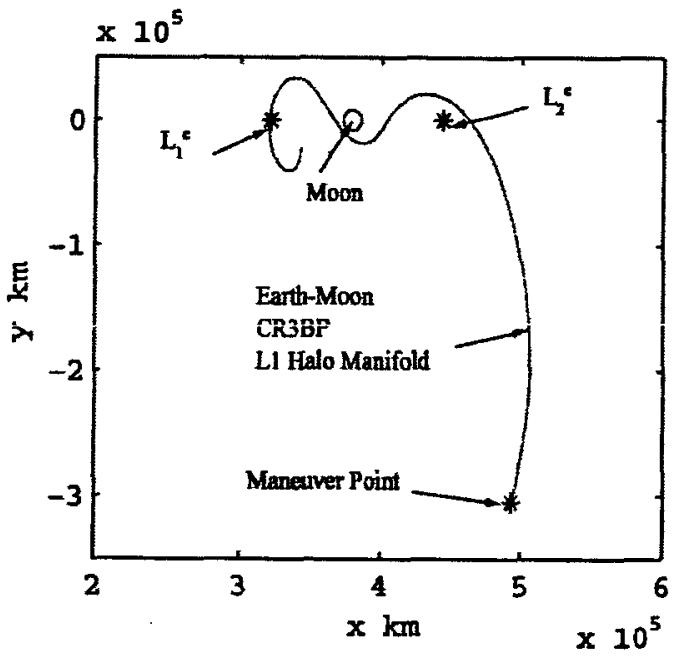

(a)

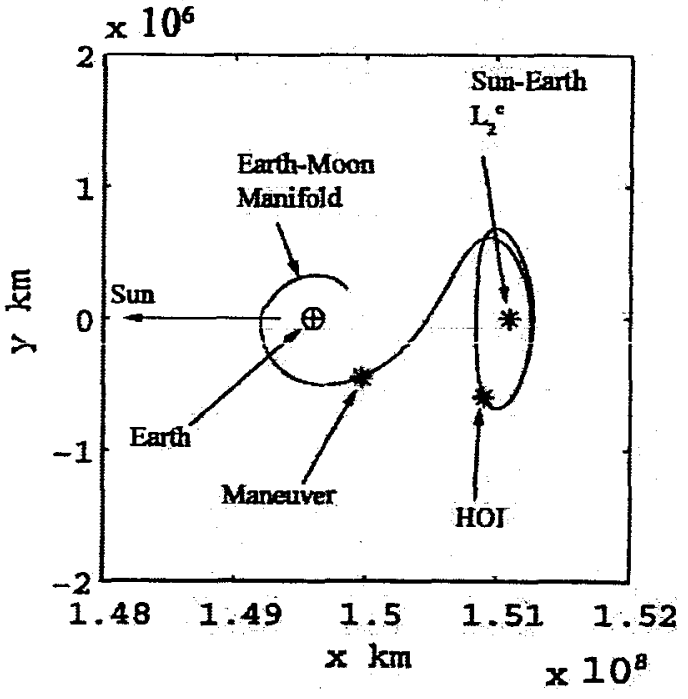

(b)

Figure 10 Transfer from the Earth-Moon $L_{1}$ Halo Orbit to a Sun-Earth $L_{2}$ Halo Orbit

Table 2

Solution for Transfer to Halo

Approximation Result at Best Intersection Point
Destination

Halo

$\mathrm{Az}=123,265 \mathrm{~km}$

Total $\Delta \mathbf{V}$ at intersection

$21.146 \mathrm{~m} / \mathrm{s}$

$3.372 \mathrm{~m} / \mathrm{s}$ 
Recall that the $\Delta \mathbf{V}$ here is calculated in the Sun-Earth frame with no regard for the dynamic effects of the Moon. This solution is transferred into a model with a complete ephemeris formulation. The Generator 3.0.2 software, developed at Purdue, incorporates an RK 8/9 integrator; the gravity of the Sun, Earth, and Moon is included for this example as well as the modeling for their ephemeris locations, but no solar radiation pressure force is added. The first arc of the transfer, as viewed in the Earth-Moon frame, appears the same as that in Figure 10(a). The Sun-Earth view is seen in Figure 11 and some differences relative to the solution in the circular problem now appear. The cost at the intersection point is now $15.27 \mathrm{~m} / \mathrm{s}$; there is no longer any libration orbit insertion $\Delta \mathbf{V}$.
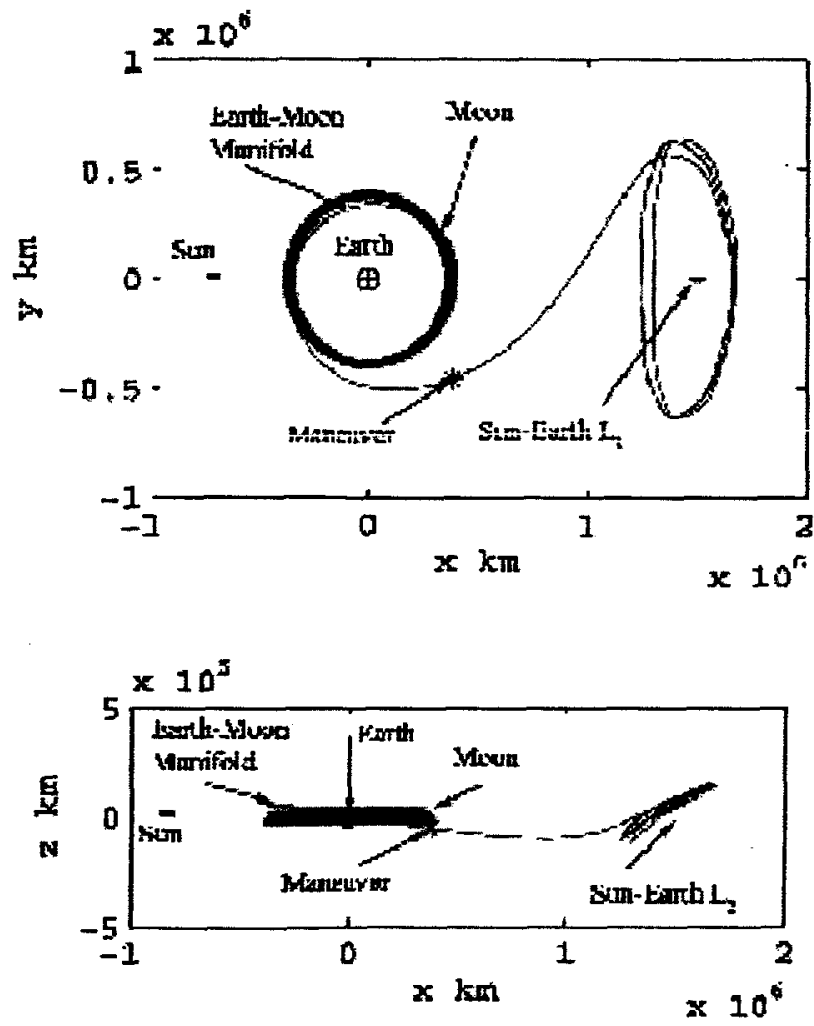

Figure 11 Transfer in the Sun-Earth System: Ephemeris Model

Finally, patch points are introduced into Generator (as marked in Figure 12) and all legs are simultaneously corrected to yield a zero cost solution. Of course, the final libration point orbits are not periodic but are Lissajous quasi-periodic trajectories. The final size of the halo orbits in the full model in the Earth-Moon system corresponds to $A z=39,600 \mathrm{~km}$; in the Sun-Earth system the out-of-plane amplitude is $\mathrm{Az}=163,000 \mathrm{~km}$. In the Sun-Earth view, the final ephemeris zero cost solution cannot be distinguished from the plot in Figure 11. It is interesting to compare the Earth-Moon departure path in the approximation in the circular problem to the final ephemeris corresponding to the no cost solution. This comparison appears in Figure 13. All of the general characteristics are maintained. As this example is reviewed, observe that every step of the process 
yields a libration point orbit of different size. This flexibility appears to aid the search for a solution.

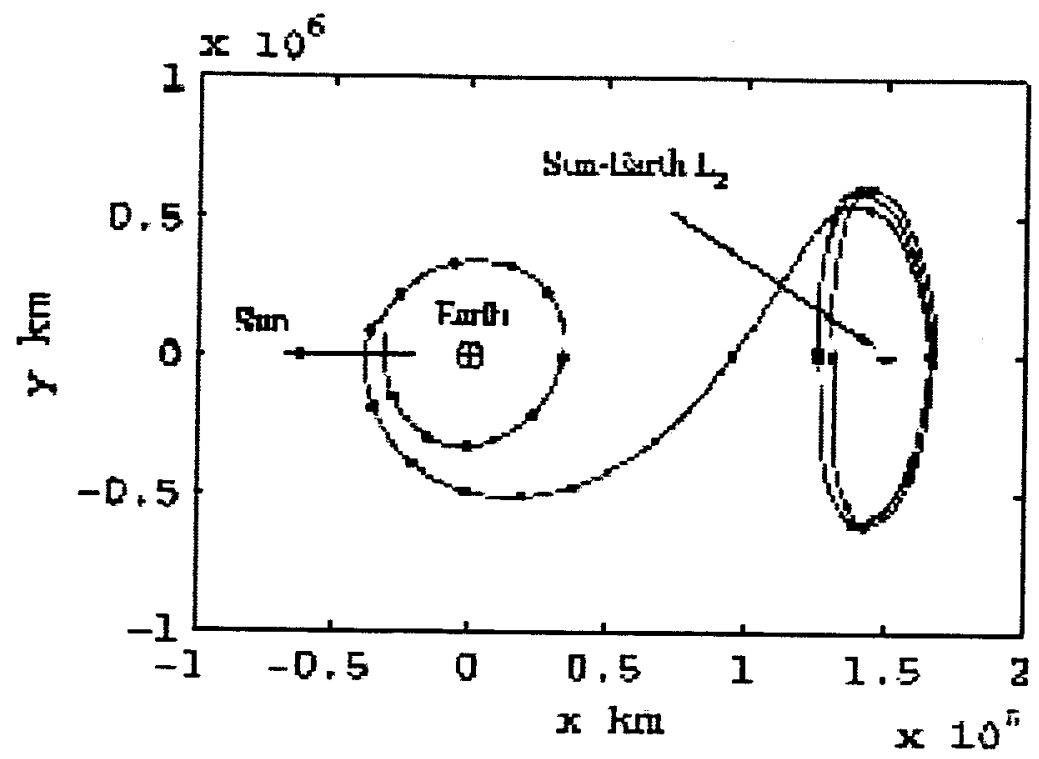

Figure 12 Patch Points in the Cost Reduction Algorithm

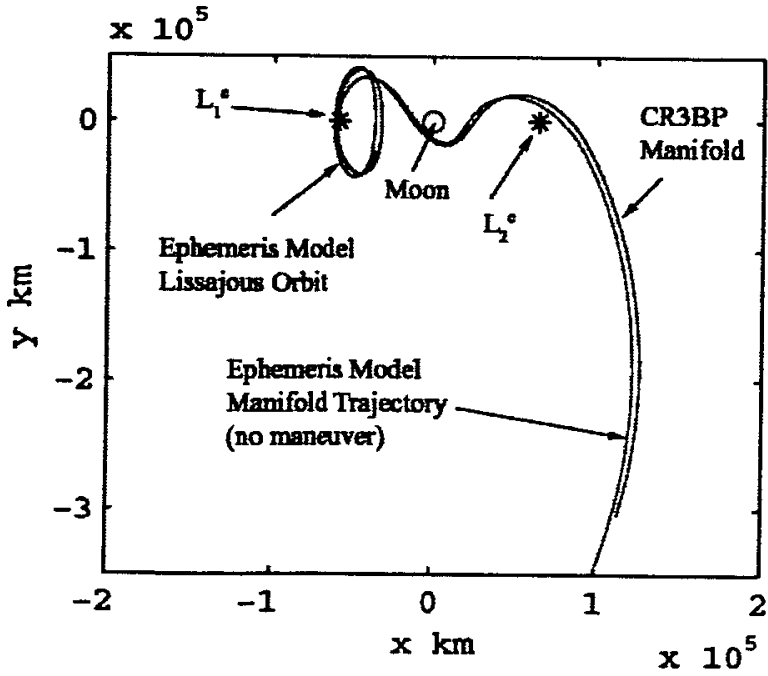

Figure 13 Comparison of the Circular Approximation to the Departure trajectory from the Earth-Moon System with the Final Ephemeris Solution 


\section{SUMMARY}

The objective of this research activity is to investigate approximations for stable and unstable manifolds to use these objects in mission design applications. Both splines and cell structures are investigated and prove successful in computations. Further developments are planned that include a wider range of applications.

\section{ACKNOWLEDGMENTS}

Portions of this work were supported by NASA and the Goddard Mission Services Evolution Center under contract numbers NCC5-358 and NCC5-727.

\section{REFERENCES}

1. G. Gómez, W. Koon, M. Lo, J. Marsden, J. Masdemont, and S. Ross, "Connecting Orbits and Invariant Manifolds in the Spatial Three-Body Problem," 2001.

2. D. Hobson, "An Efficient Method for Computing Invariant Manifolds of Planar Maps," Journal of Computational Physics, Vol. 104, 1993, pp. 14-22.

3. R. Guder, M. Dellnitz, and E. Kreuzer, "An Adaptive Method for the Approximation of the Generalized Cell Mapping," Chaos, Solitons, \& Fractals, Vol. 8, No. 4, 1997, pp. 525-534.

4. M. Dellnitz and A. Hohmann, "A Subdivision algorithm for the computation of unstable manifolds and global attractors," Numerische Mathematik, Vol. 75, 1997, pp. 292-317.

5. B. Krauskopf and H. Osinga, "Growing 1D and Quasi-2D Unstable Manifolds of Maps," Journal of Computational Physics, Vol. 146, 1998, pp. 404-419.

6. B. Krauskopf and H. Osinga, "Globalizing Two-Dimensional Unstable Manifolds of Maps," International Journal of Bifurcation and Chaos, Vol. 8, No. 3, 1998, pp. 483-503.

7. K.C.,Howell and J.P. Anderson, "Triana Contingency Analysis: Final Report," IOM AAE0140-006, prepared for NASA Goddard Triana Flight Project, May 2001.

8. J. Anderson, "Earth-to-Lissajous Transfers and Recovery Trajectories Using an Ephemeris Model," M.S. Thesis, School of Aeronautics and Astronautics, Purdue University, 2001.

9. R.W. Farquhar, D.W. Dunham, Y. Guo, and J.V. McAdams, "Utilization of Libration Points for Human Exploration in the Sun-Earth-Moon System and Beyond," 54 $4^{\text {th }}$ International Astronautical Congress, Bremen, Germany, October 2003, Paper No. IAC-03-IAA.13.2.03.

10. D.C. Folta, M. Beckman, S.J. Leete, G.C. Marr, M. Mesarch, and S. Cooley, "Servicing and Deployment of National Resources in Sun-Earth Libration Point Orbits," $53^{\text {rd }}$ International Astronautical Congress, The World Space Congress, October 2002, Houston, Texas, Paper IAC-02-Q.6.08. 
11. M.W. Lo and S.D. Ross, "The Lunar $\mathrm{L}_{1}$ Gateway: Portal to the Stars and Beyond," AIAA Astrodynamics Conference, Albuquerque, New Mexico, August

12. W.S. Koon, M.W. Lo, J.E. Marsden, and S.D. Ross, "Shoot the Moon," AAS Paper No. 00166.

13. W. Koon, M. Lo, J. Marsden, and S. Ross, "Constructing a Low Energy Transfer Between Jovian Moons," Contemporary Mathematics, Vol. 292, 2002, pp. 129-145.

14. G. Gómez, W. Koon, M. Lo, J. Marsden, J. Masdemont, and S. Ross, "Invariant Manifolds, the Spatial Three-Body Peoblem, and Space Mission Design," AAS/AIAA Astrodynamics Specialist Conference, Quebec City, Paper No. AAS 01-301, 2001. 